\title{
ISS emergency scenarios and a virtual training simulator for Flight Controllers
}

\author{
Thomas Uhlig a,*, Frank-Cyrus Roshani ${ }^{\mathrm{a}}$, Ciro Amodio ${ }^{\mathrm{a}}$, Alessandro Rovera ${ }^{\mathrm{b}}$, \\ Nikola Zekusic ${ }^{\mathrm{c}}$, Hannes Helmholz ${ }^{\mathrm{d}}$, Matthew Fairchild ${ }^{\mathrm{a}}$ \\ a Deutsches Zentrum für Luft-und Raumfahrt, German Space Operations Center, Münchner Str. 20, 82234 Wessling, Germany \\ ${ }^{\mathrm{b}}$ ALTEC S.p.A., Corso Marche 79, 10146 Torino, Italy \\ ${ }^{c}$ LSE SPACE GmbH, Argelsrieder Feld 22, 82234 Wessling, Germany \\ d Technische Universität Berlin, Audio Communication Group, Einsteinufer 17c, 10587 Berlin, Germany
}

\section{A R T I C L E I N F O}

\section{Article history:}

Received 11 January 2016

Accepted 4 August 2016

Available online 5 August 2016

\section{Keywords:}

Human spaceflight

Flight Control Team

Emergency

Mission control

Simulation

Virtual reality

\begin{abstract}
A B S T R A C T
The current emergency response concept for the International Space Station (ISS) includes the support of the Flight Control Team. Therefore, the team members need to be trained in emergencies and the corresponding crew procedures to ensure a smooth collaboration between crew and ground. In the case where the astronaut and ground personnel training is not collocated it is a challenging endeavor to ensure and maintain proper knowledge and skills for the Flight Control Team. Therefore, a virtual 3D simulator at the Columbus Control Center (Col-CC) is presented, which is used for ground personnel training in the on-board emergency response. The paper briefly introduces the main ISS emergency scenarios and the corresponding response strategy, details the resulting learning objectives for the Flight Controllers and elaborates on the new simulation method, which will be used in the future. The status of the 3D simulator, first experiences and further plans are discussed.
\end{abstract}

(c) 2016 IAA. Published by Elsevier Ltd. All rights reserved.

\section{Introduction}

Operation of a manned platform in space like the International Space Station (ISS) requires concepts for emergency cases [1]. The procedures which are available for those cases are designed for maximum crew autonomy to avoid critical dependencies on communication equipment and hence on the link to Mission Control. However, in the Low Earth Orbit (LEO) regime with neglegtable communication delays the Mission Control Center can still provide a valuable support to the emergency response of the on-board astronauts.

For that reason not only the astronauts need to be extensively trained in the various emergency response strategies, also the Flight Control Teams need training in it.

The first level here is classroom training, which conveys the theoretical knowledge and contains first walk-throughs of the procedures.

The second level is simulations, during which the Flight Control Team is put into a real control room in a real operational context with simulated data stream and commanding with trainers roleplaying the astronauts, who read down a pre-scripted scenario.

\footnotetext{
* Corresponding author.

E-mail address: thomas.uhlig@dlr.de (T. Uhlig).
}

The difficulty here is that the trainer is in many cases not the expert in the on-board emergency handling, since they are in some setups not involved in the corresponding crew training, so imitating the crew during the simulation is difficult; and involving a real astronaut in the simulations is often difficult due to their busy schedules.

At the Columbus Control Center (Col-CC) in Oberpfaffenhofen/ Germany, one of the five main control centers for the ISS [2], a novel virtual reality simulator was developed for emergency simulations, which allows one to also "play" the crew part of the scenario. With that approach, the "crew" can also be played by a corresponding Flight Control Team member, which increases the fidelity of the simulation, since a proven expert in the emergency field is now also involved in the "on-board part". For that person it has the additional training effect that also the crew part of the emergency is reinforced and an even better and more integrated understanding of the response strategy is acquired.

\section{ISS emergency response}

Beside medical emergencies [3], which are of low relevance for the Flight Control Team due to the fact that they are not directly involved, there are three major technical emergencies defined for the International Space Station. A fire can directly endanger the 
lives of the crew [4]. A depressurization due to e.g. a micrometeoroid or orbital debris strike can lead to a perforation of the station's shell and can cause a loss of the vital air. The atmosphere on-board can be poisoned by the release of toxic substances, e.g. in case ammonia used in the external cooling loop enters the internal cooling water system due to a rupture of an interface heat exchanger and is subsequently ending up in the cabin air.

For all emergencies the crew is extensively trained to be able to immediately execute from memory the so-called "Common Emergency Response": As an initial step everybody's attention needs to be drawn to the dangerous situation. For that, the ISS wide alarm system is activated. This is either done automatically, in case the vehicle's sensors have detected the emergency condition (smoke detectors, pressure sensors, pressure increase in the cooling water loop), by a Flight Controller observing emergency clues in their telemetry or by the crew pressing the emergency button (visible smoke, burning odor, detected air pressure drop, observed toxic spill or other signatures).

The ISS wide alarm ("Caution and Warning" subsystem) is also broadcasted to all ISS control centers and also causes an emergency alarm message to be released, providing some level of detail (class of emergency, location, failed equipment) to the Flight Control Team. The alarm message might be accompanied by voice communications with the crew.

The crew then gathers and establishes a so-called safe haven, essentially a zone in which it is safe to stay, has the required emergency response equipment, a computer to command the space station and a clear exit path to the Soyuz spacecraft, which act as rescue ships of the space station. It is then at the commander's discretion to decide if the crew either starts with the emergency specific response procedures [5], or immediately evacuates the station.

The station control software will simultaneously take action. Depending on the emergency case and its initiation (manually or automatically) it executes various actions which aim at two main objectives: to stop/limit the source of the emergency and its propagation and to assist the crew in their response strategies.

The personnel in the control rooms stop all current operations immediately and fully focus on the emergency case. The lead is either with the Mission Control Houston or with the Moscow Control Center, depending on the on board location of the emergency. After that the main tasks of the flight control teams are to check the automatic software response of the space station, the search for indicators in case the emergency source is unknown and finally the support of the crew, who is of course prime in the emergency response.

In the following sections the detailed strategies reflected in the procedures in response to an on-board ISS emergency will briefly be described, sorted by cases. Before doing that, it's important to keep in mind that the priorities followed by the real time operating policy are: Crew safety, vehicle safety and mission success. These priorities are emphasized and further detailed in the strategies developed for the response to an on-board emergency event.

\subsection{Fire Emergency}

After the execution of the Common Emergency Response, the emergency annunciation (if not yet done) and the determination of air quality and a fire classification is done. All these steps are part of the Initial Response, as depicted in Fig. 1.

For the classification step, two cases can be distinguished: In case there are visible flames or smoke, the air quality per definition is considered poor and the crew is immediately required to don the Portable Breathing Apparatus (PBA) or the equivalent Russian equipment, which constitute an independent oxygen

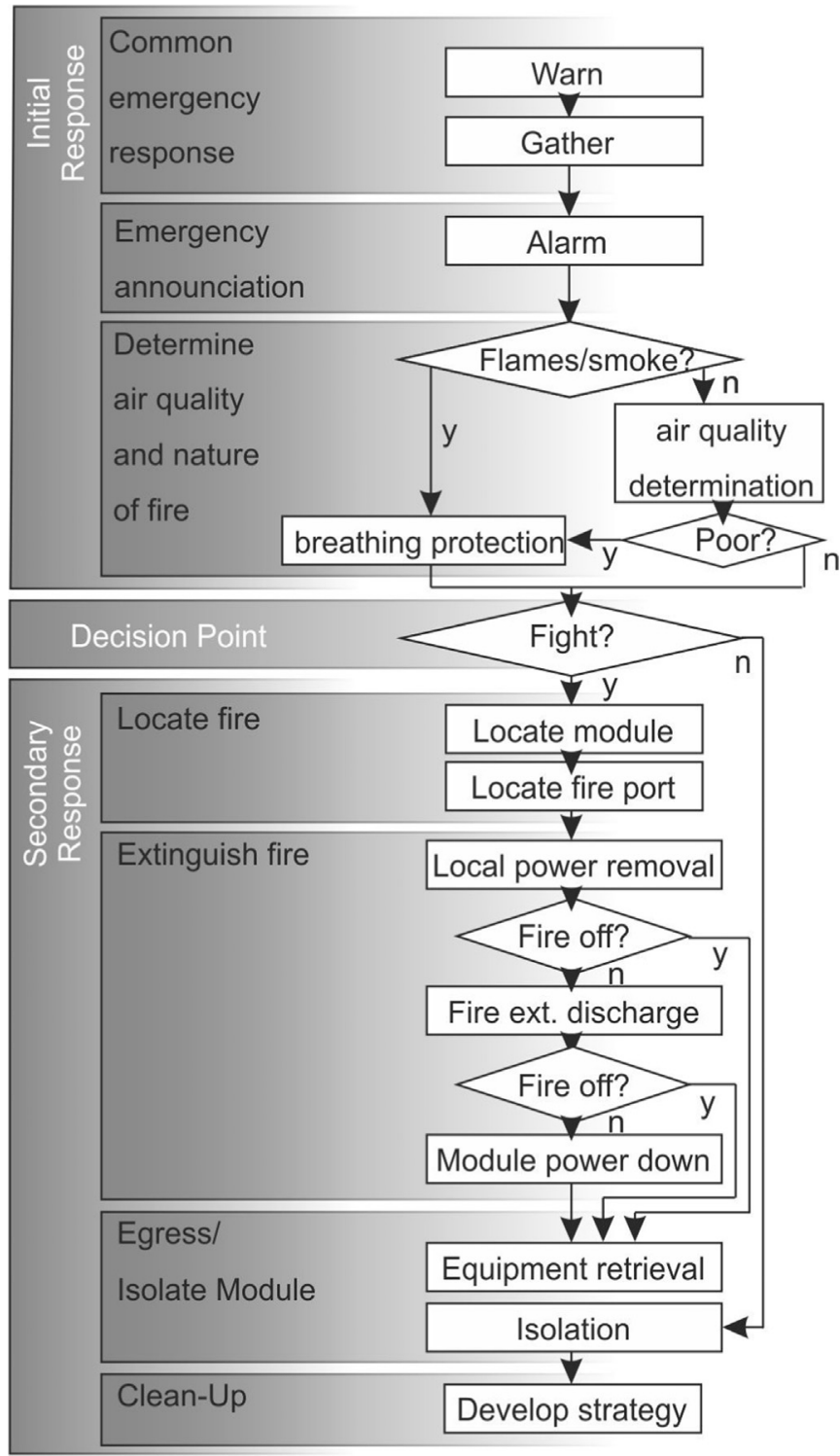

Fig. 1. The ISS fire strategy (from ISS emergency books, not publicly accessible).

supply for the crew and are available all over the space station. In case of no visible clues of the ongoing fire, crew is required to utilize dedicated Compound Specific Analysers (CSA) for Combustion Products (CP) to determine the quality of the air. In case certain limits for fire byproducts are exceeded, crew is again required to wear breathing protection.

If after the initial response crew is considered safe for the moment, then they can decide about the secondary response.

During the secondary response crew changes from PBAs to dedicated Emergency Masks. These masks do not provide oxygen, but filter the surrounding air. They are only available in a central repository in the Russian segment, but have a much longer expiration duration than the PBAs.

The first step in the secondary response is the actual search for the fire location. Here, various clues are used: Sensory (odor smoke concentration or direction), telemetry indications (tripped equipment, increased temperature), warning lights. In addition again the CSA-CPs provide measurements for each module and following increasing values leads to the suspected module. In the identified module, the crew uses dedicated probes on the CSA-CPs, which are inserted in the fire ports. These are distributed all over 
the module and allow access behind the various panels and to the inside of racks.

After identification of the location, the fire needs to be extinguished. A hierarchical approach is followed: First step is the removal of power to the affected equipment or area. Next level is the usage and discharge of the $\mathrm{CO}_{2}$ fire extinguisher if required. In that case crew has to switch from the Emergency Masks to PBAs again due to the increased $\mathrm{CO} 2$ level after the discharge. If the fire is still not eliminated (criteria: stable or decreasing CSA-CP readings), the module is fully or partially powered down.

After that the next step is the isolation of the affected module. In case the crew still feels safe to enter the module, they will attempt to retrieve important equipment parts before the hatch is closed. It is important to be aware that the module might be dark after a potential complete power-down, crew is then guided by the phosphorescing Emergency Egress Guidance System (EEGS). Finally various post-fire clean-up strategies will be discussed.

\subsection{Rapid Depress}

The strategy developed for the response to a Rapid Depress event takes into account the number of crew members who are on board: If only $3 \mathrm{crew}$ members are on-board, the strategy takes into account the docked location of their Soyuz. In the case of 9 crew members 3 take care of the leak check of their Soyuz while the rest is applying the 6 crew member strategy described hereafter.

As for the previous emergency event the response strategy can be split in two parts, as shown in Fig. 2: an "Initial Response" whose steps deal with saving the crew and a "Secondary Response" for the vehicle and mission safety. The first step is the memorized Common Emergency Response, which is executed by the crew.

Next step is then to perform pressure measurements and to determine the leak rate and hence the time until critical pressure values are reached, the so-called Reserve Time (T.res). For this task the crew relies on robust mechanical handheld Russian equipment (Manovacumeter) to measure the absolute pressure, and on graphs in the emergency books which correlate the initial pressure and the duration of a pressure drop by $1 \mathrm{mmHg}$ with the expected Reserve Time.

The absolute pressure value dictates whether protection equipment (the already mentioned PBAs) needs to be worn by the crew. The Reserve Time determines the time left for the actual fighting of the route cause: a pressure lower than $527 \mathrm{mmHg}$ requires the crew to wear PBAs, a remaining T.res $<10 \mathrm{~min}$ demands an immediate isolation of the leak to a segment or in the worst case the crew needs to evacuate the station.

As a last step of the Initial Response the Soyuz spacecraft, which would function as rescue vehicles, and the corresponding adjacent modules (one of the Russian Mini-Research Modules, MRMs) will be leak checked. This is done by the corresponding Soyuz crews in parallel using the so-called tap technique: the hatch is closed and briefly pressed against its sealing. If released it either automatically opens or it remains closed, dependent on the pressure gradient between both sides. In case no immediate result can be derived the hatch is closed for a longer period of time and the same effect observed. In case the MRM/Soyuz is the leaking compartment, the ISS itself is considered as healthy and more analysis will follow on the Soyuz side.

In case MRM/Soyuz is not leaking, crew can enter the Secondary Response, as shown in Fig. 2, always keeping in mind the remaining Reserve Time stays over $10 \mathrm{~min}$.

While the check of the MRM/Soyuz integrity is ongoing, air flow sensors in the Russian segment perform an analysis about the air stream direction at all Russian hatches. In the ideal case these measurements provide indications whether the Russian segment

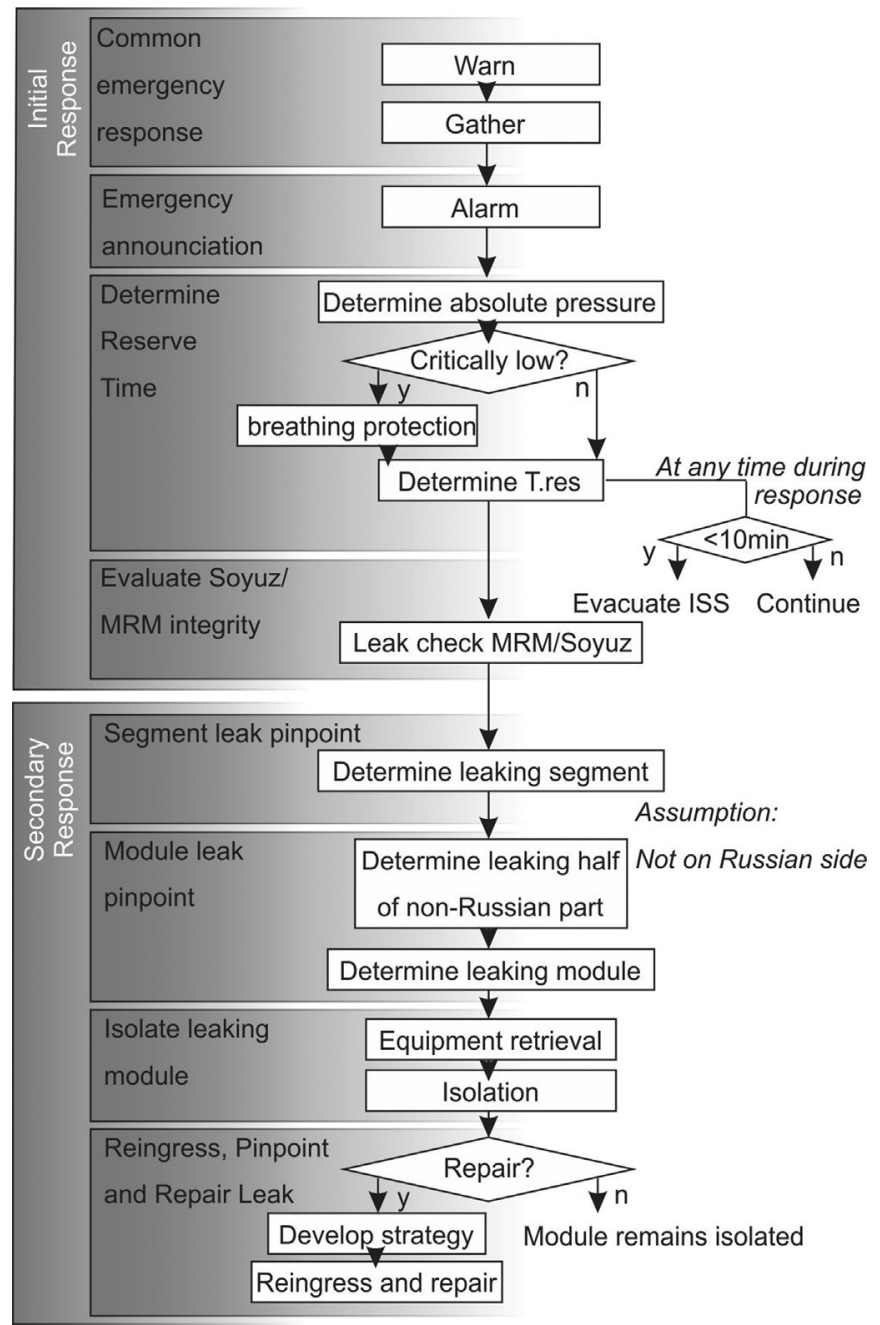

Fig. 2. The ISS depress strategy (from ISS emergency books, not publicly accessible).

or even which module is leaking or not.

In case the measurements do not provide clues which segment is perforated, the crew has to manually determine that in the first part of the Secondary Response. This is done by closing the ГAPMA1 hatch (see Fig. 3) and hence isolating the Russian segment from the American segment including the connected Japanese/ European modules. Then the above mentioned tap technique and pressure measurements in the Russian segment (crew always stays on the Soyuz $=$ escape side of the corresponding hatch) indicate the leaking segment.

Since the target group for our training are European Flight Controllers whose involvement in Russian segment leaks is marginal we only focus here on cases with a leak in the non-Russian part of the ISS. Having determined the American (Japanese/European) segment as cause for the pressure decrease, crew would now enter the next part of the Secondary Response strategy: The Module Leak Pinpoint.

To get a rough idea, crew then closes the "Node $2 \mathrm{Aft}$ " hatch in order to divide the American (Japanese/European) segment by half to determine which part is the leaking one. Based on the result, they continue to investigate that part using the strategy to start with the module which is farthest away from the Russian Segment/the Soyuz, axial modules (first Japanese, then European) first, then moving "backward". The corresponding module is checked by closing the hatch and measuring the pressure on the "escape side" of the hatch: Should the pressure remain steady, then 


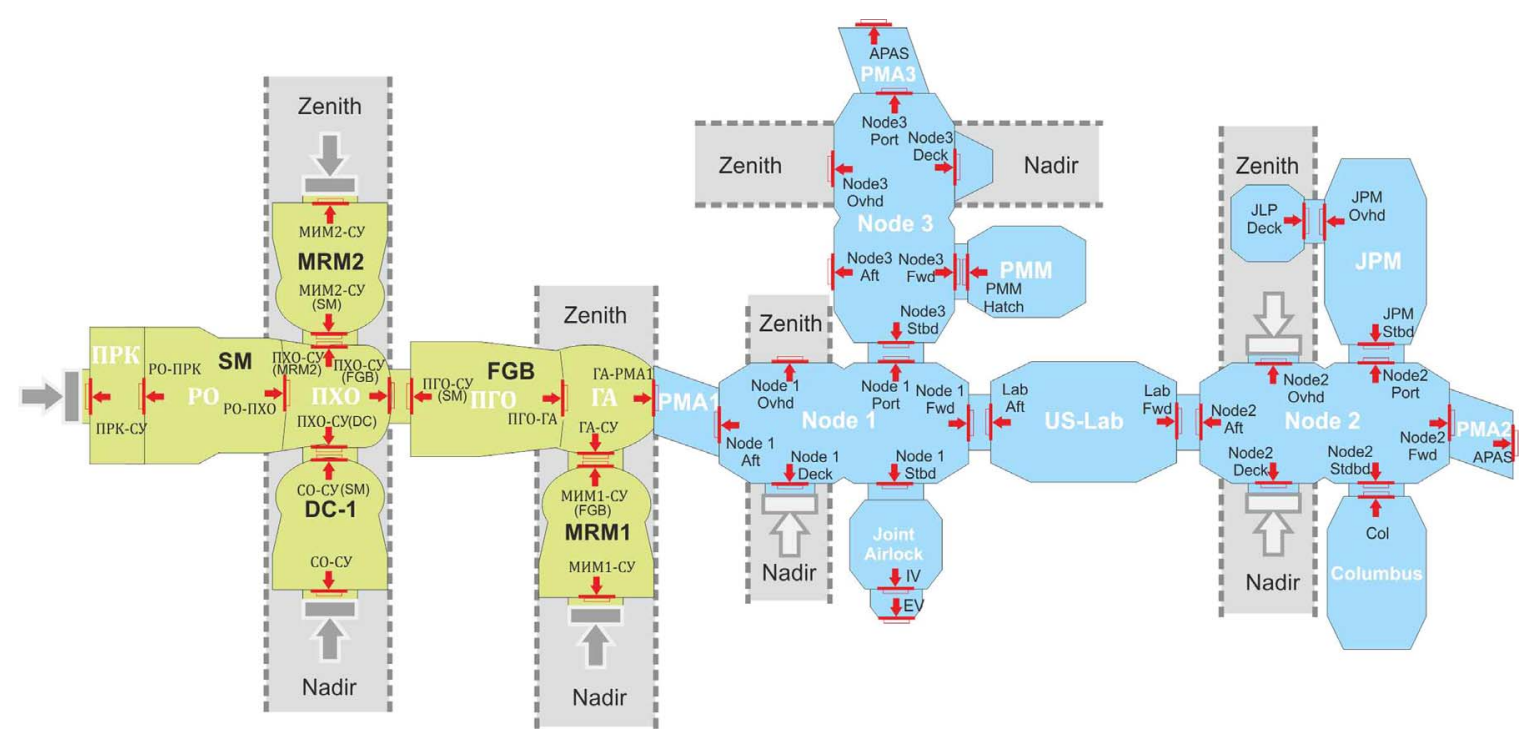

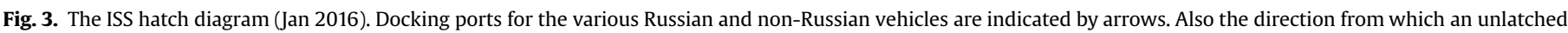

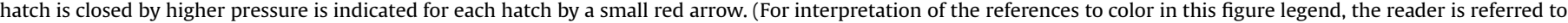
the web version of this article.)

the leaking module is found. If enough time is remaining, then the crew retrieves important equipment prior to isolating the module (s), since it is likely that the modules cannot be entered for a prolonged time. Since crew will always stay on the Soyuz side of the leak, all modules forward of the leaking one are also no longer accessible, the corresponding hatches remain closed to conserve the atmosphere in them.

Once the leak has been isolated and crew is safe, the emergency event ends. Nevertheless there might be still the opportunity to re-ingress and repair the leaking module. As a last step of the Secondary Response crew might then decide - based on the Reserve Time, the expert recommendations and crew safety whether a re-ingress and repair is an option. There are various ways to seal a leak which are all available on board in a dedicated ISS Leak Kit. This also contains an Ultrasound detector to locate the acoustic signature of the escaping air [6]. Also telemetry indications (equipment damaged by an impact) or the vehicle attitude changes (escaping air generates a torque) might provide clues.

\subsection{Toxic atmosphere}

A spill of toxic material scenario is currently not implemented in the 3D emergency simulator, since the corresponding crew responses are of less critical nature, do not require extensive interactions with the control centers and are not spread over the entire vehicle. However, for completeness the toxic spill response shall also be briefly described here.

Since the reaction is completely different in case of an ammonia release we will treat the latter as a separate case, although they are usually considered as one.

\subsubsection{Non-ammonia toxic spill}

The Initial Response for a non-ammonia contingency consists of the Common Emergency Response and the annunciation of the emergency which is of particular importance here, since there are no on-board mechanisms for detection. The annunciation consists of pressing the corresponding emergency alarm button together with a previous voice message to ground that the case does not involve ammonia.

The Secondary Response consists of the determination of the hazard level of the spilled substance. There are five levels $(0-$ nonhazardous to 4 - most hazardous) defined for the ISS, and every substance on board is classified and labeled accordingly [7]. In case of an unknown substance, certain rules are applied based on the containability and the state (gaseous, liquid, solid) to define a "working hazard level".

Based on the determined hazard level the appropriate personal protection means are donned in the next level of the Secondary Response. As final step a clean-up is attempted, for which various devices are available on board.

\subsubsection{Ammonia release}

Ammonia is used in the external American cooling system of the ISS, which interfaces with the water-based internal cooling circuits. Therefore intrusion of ammonia is one of the emergency scenarios and since it is highly lethal, an immediate response is required [8]. Basically crew immediately escapes to the Russian part of the ISS, closes the Node 1 aft hatch (see Fig. 3), remove their clothes and leaves them in the PMA to minimize ammonia transfer, enters the Russian segment and also closes the hatch to the PMA. In the Russian segment dedicated Emergency Masks with ammonia cartridges are donned by the crew and measurements are taken to ensure clean atmosphere there. Based on the results, the crew must decide on whether they can safely stay in the Russian segment or need to evacuate the ISS.

\section{Training requirements and conditions}

The training of astronauts $[9,10]$ on emergency responses is imperative due to their autonomous and central role. However, Flight Controllers also have emergency training elements in their certification flows [11]. For European Flight Controllers there is currently an Emergency classroom training available in which the trainees are introduced into the crew actions in an emergency case, in the Columbus automatic reaction and their role in such situations. The training content is defined via Learning Objectives according to the concept of Instructional System Design [12]. The Learning Objectives in this training related to the crew actions on a fire and rapid depress emergency which are hence in scope of the simulator, are listed in Table 1.

According to Bloom's taxonomy [13] all of them are on the Comprehension level of the cognitive domain, which is in line with the future task of a flight controller with regards to the crew 
Table 1

Crew action related Learning Objectives for the emergency class room training for Flight Controllers.

1. Describe the strategy behind the ISS Fire Emergency Responses

2. Describe the functions, use and constraints of hardware/tools used in the ISS Fire Emergency Response

3. Describe how a 6 person crew responds to an ISS Fire Emergency

4. Describe ISS Emergency Equipment used in Rapid Depress Responses

5. Describe the strategy behind the Rapid Depress Response

6. Summarize the Rapid Depress response dependent upon the ISS Crew complement

7. Select the procedures used in a Fire/Rapid Depress Release Response

emergency actions: They do not have to execute the crew response by themselves (which would justify the Application level), but just have to have a broad understanding of what the crew is doing in order to better interact and assist them. Therefore training in the past had consisted of classroom lectures and theoretical walkthroughs through the emergency crew procedures.

According to the concept "learning by doing" [14] an increased learning efficiency is observed where the trainee is directly with working the procedures and flows, which he/she needs to memorize. Therefore the approach to put a Flight Controller into the position of an astronaut and let her/him execute the emergency procedures in a 3D virtual environment seems to be promising. It will be used in future training sessions to enhance the classroom lessons and replace the procedure walkthroughs.

A second training element for Flight Controllers with regards to emergency operations consists of dedicated emergency simulations or normal simulations, which are ended with an emergency scenario. During these events in the past a European astronaut was "paper-simulating" an on-board crew member, the Flight Control Team was placed into the training control room, the reactions of the space station were mimicked by a software simulator and an instructor team was surrogating the Houston team. Due to the high complexity of the scenario and the lack of detailed knowledge of the partner's actions the entire simulation was based on a pre-prepared script, so deviations are almost impossible and the "real emergency books" are not used on the instructor's side of the event. This concept had some additional weaknesses: The availability of "real" astronauts for simulations was very limited and hence an additional major constraint in the already complex scheduling. Their emergency training was sometimes outdated, if they were not assigned mid-term to a mission (and if they were, they were no longer available for simulations) and a good Flight Controller training opportunity (to play the crew) was given away.

The Learning Objectives for these emergency simulations, which are directly linked to the crew performance on board, were extracted and pasted in Table 2.

Of course for these simulation scenarios the usage of a virtual 3D simulator in the future is considered beneficial in several aspects: A Flight Controller can play the crew and hence is immerged into the emergency procedures and derives a very broad understanding on the crew actions. In addition his expert knowledge

Table 2

Crew action related Learning Objectives for the emergency simulation training for Flight Controllers. also allows a simulation which is not only scripted, but can be more dynamic.

\section{Simulator realization}

\subsection{Basic considerations}

Although under normal circumstances there are either three or six astronauts on board, for the first implementation of the virtual training simulator it was decided to follow a one player setup only. If we assume a three persons crew, one astronaut would then stay in safe haven and control the ISS systems via a laptop, whereas the two colleagues - according to the buddy system - would move forward and tackle the emergency situation. As per procedure there is no mandatory interaction or job sharing foreseen between those two, therefore it is a fair assumption that only the trainee is acting and he/she is silently (and invisibly) shadowed by the second astronaut.

It was also decided to follow a kind of "mixed reality" approach: The real world should not be blocked out completely as would be the case e.g. by using a Head Mounted Display (HMD). Rather the trainee should be able to interact also with "real world items" during the simulation so that e.g. the printed versions of the emergency crew procedures can be used in parallel without having to use means of Augmented Reality. With that it is ensured that the trainee better gets acquainted with the structure of these procedures, which he also needs to use on console, an electronic implementation of the procedures for the virtual world (and subsequent updates of those, which would be additional effort) can be avoided and the training team can rely on the already existing in-house configuration control processes to ensure that the latest version is always used.

\subsection{Hardware}

At the German Space Operations Center where the Col-CC is located a so-called CAVE (Cave Automatic Virtual Environment) is installed. It consists of 5 semi-transparent flat screens horizontally angled at $135^{\circ}$ to each other so that they form a $180^{\circ}$ surround screen system that surrounds the user(s), as depicted in Figs. 4 and 5. Ten projectors located behind the screens as seen from the human participant project their images onto the screens, thus enabling stereo back projection by use of linear polarization to separate the images for the left and right eye respectively. In the current set-up each projector is connected to its own vision system PC, the so-called client PC which renders one channel of the stereo image pair for the respective portion of the whole $180^{\circ}$ scene view. The vision system PCs are complemented by two additional PCs: the master and the server PC. The master PC handles the user's inputs, controls the simulation application and carries

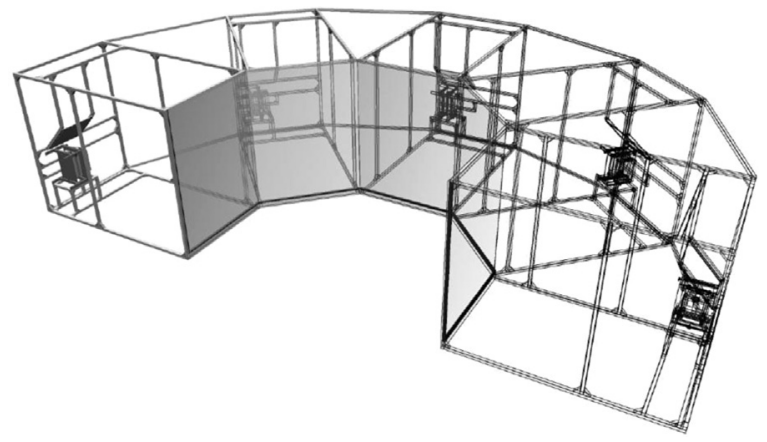

Fig. 4. CAVE System Layout at GSOC.

\footnotetext{
1. Demonstrate awareness of on-board support equipment usage

2. Maintain awareness of crew emergency strategies and actions

3. Coordinate - and if the situation dictates - communicate effectively between the crew and ground

4. Demonstrate awareness of crew health/safety during an emergency situation
}

\section{(1)}

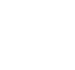




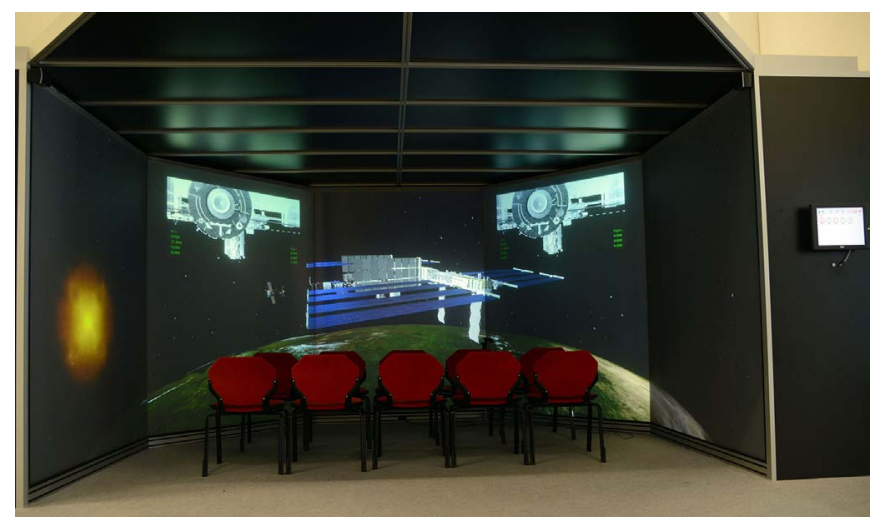

Fig. 5. CAVE system at GSOC in use (from [15]).

out the synchronisation between the client PCs. The user can interact with the running simulation application by use of a conventional Gamepad which is connected to the master PC via a USB cable connection. The server PC acts as a file server on which the actual executable files of the simulation programs are located and made available to all other PCs via disc mounting. The server PC also acts as the central control entity for the whole system, with which the operator can start up and shut down the computers and the projectors or choose which simulation application to run. For this the server PC is connected to a touch screen monitor at the front side of the CAVE system on which the Graphical User Interface of the control application is displayed. All computers and the projectors are connected via a local network (LAN). This system is intended to provide a Virtual Reality capability with which in general planning, preparation and execution of space projects can be supported. In recent times it was also used to demonstrate actual projects operated by GSOC like Columbus to the interested public.

\subsection{Software and implementation}

Unlike most other applications in the area of training, simulation and scientific visualization, the virtual ISS emergency scenario simulation was developed with the help of a commercial game engine (in this case Unity) as the development environment. The main reason for this decision was that development of the simulation application would mainly be done by bachelor and masters students, interns and student trainees who not necessarily would have prior experience in common graphics APIs or frameworks like OpenGL. Unity provides an easy-to-learn development environment with a user interface which is oriented more towards usual 3D graphics programs. Its ease-of-use is supported by a very active user community and a vast amount of training material and content at little or no cost. It offers the possibility to create visually appealing applications with limited programming effort all within a complete development environment including a graphical scene editor, built-in physics engine and tools for creating animations besides other valuable features. Also Unity is very popular especially among independent game developers, students and hobbyists also due to its advantageous licensing policy. In addition Unity makes it easy to deploy the applications for a multitude of devices ranging from desktop PCs, game consoles to smartphones and VR headsets like Oculus Rift and GearVR. This offers the possibility to use additional training media beside the CAVE system described here. In the future the range of training media could be expanded to include also simulation applications running on mobile devices like smartphones, tablet computers and mobile VR headsets so that Flight Controllers, but probably also astronauts can use them in nearly any location.

\subsection{Implementation of the emergency scenarios}

The first step in developing a simulation scenario with a computer game engine like Unity is to create the respective 3D content (the virtual world) with which the user should interact. In our case the virtual world consists of the interior of the ISS and includes all necessary equipment for handling the emergency cases. When developing the prototype for the emergency scenario simulator the main focus was put on the overall functionality and on supporting the learning objectives of the emergency training rather than creating a highly realistic virtual environment at first. So detailing the interior of the ISS to a level of photorealistic representation was not given a high priority since this was not considered crucial for running the emergency procedures, and would probably be more distracting than helpful. Furthermore the level of detail of the 3D objects of the virtual environment was kept relatively simple to regard the overall frame rate of the simulation. For the simulation execution a first person view was chosen, which is quite common among computer games. In this scenario the trainee assumes the role of one of the astronauts on
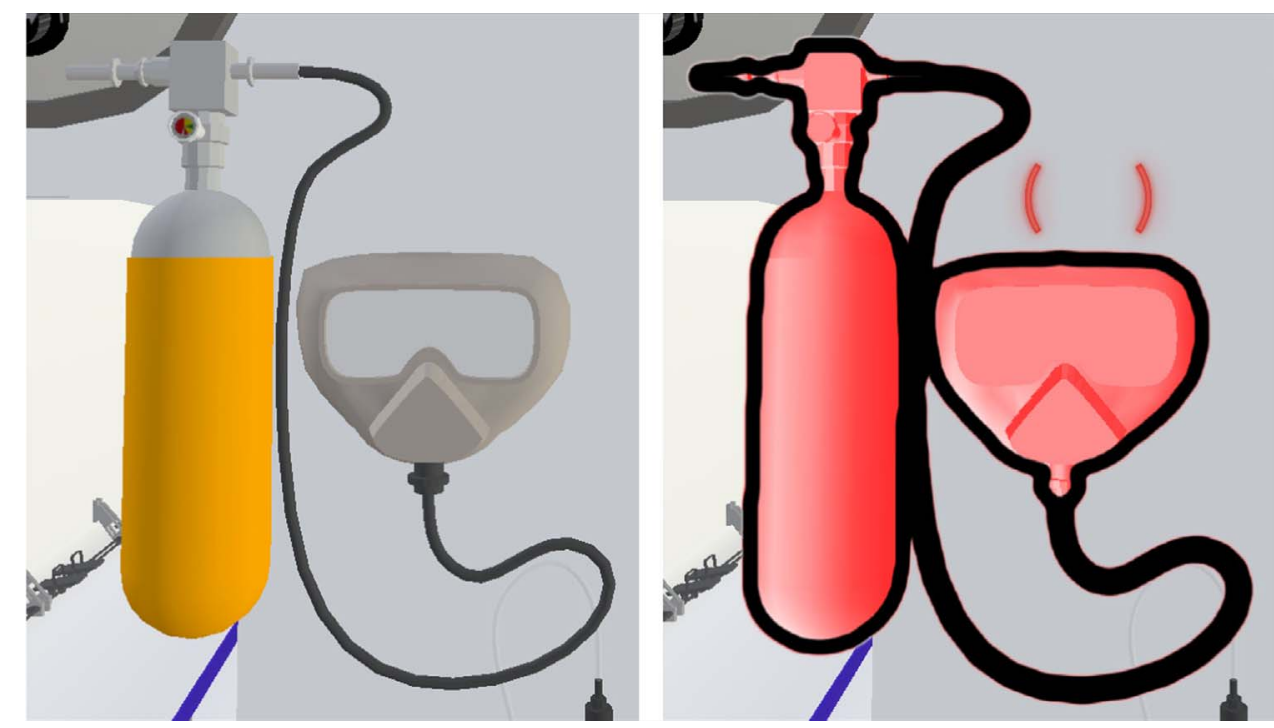

Fig. 6. The Portable Breathing Apparatus (PBA) is highlighted, before the participants can "pick it up" (from [15]). 


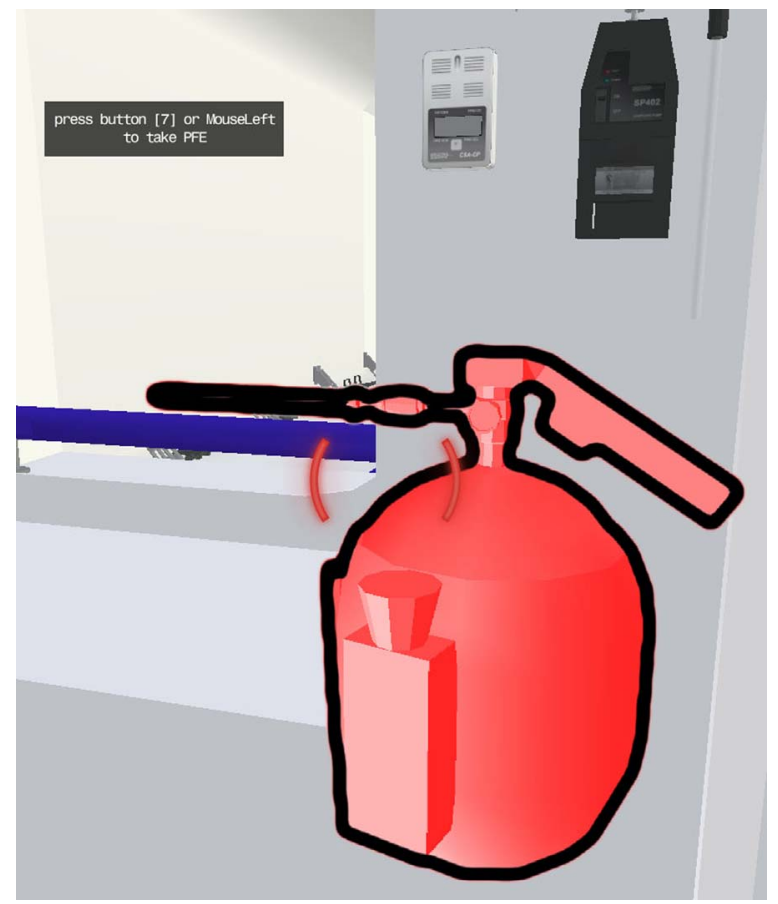

Fig. 7. Tool-tip like information (upper left) displayed when highlighting an object (from [15]).

board the ISS who would be in charge of handling the emergency situation. The trainee can navigate through the virtual space station using a Gamepad. He or she can interact with the virtual objects by pointing a bracket shaped cursor at the desired object which is then highlighted (see Fig. 6). In order to reduce eye strain in the stereo environment the spatial depth of the cursor within the virtual world is matched to the object which it is traversing thus eliminating conflicting visual depth cues. The interaction of the user with the virtual objects is further supported by tooltiplike information which is displayed next to the object the user is pointing at, as depicted in Fig. 7. This information is basically used to show the user how to interact with the object in focus. This behavior of course compromises somehow the full immersion of the user into the virtual world as this information would be a socalled non-diegetic representation [15,16], one that would not exist in the real world. But as we have laid out in Section 4.1 a full immersion of the user was not intended as he/she would have to use non-virtual equipment in parallel during the training lessons anyway.

In the same manner also a map of the ISS in which the actual position and looking direction of the virtual self is indicated by a

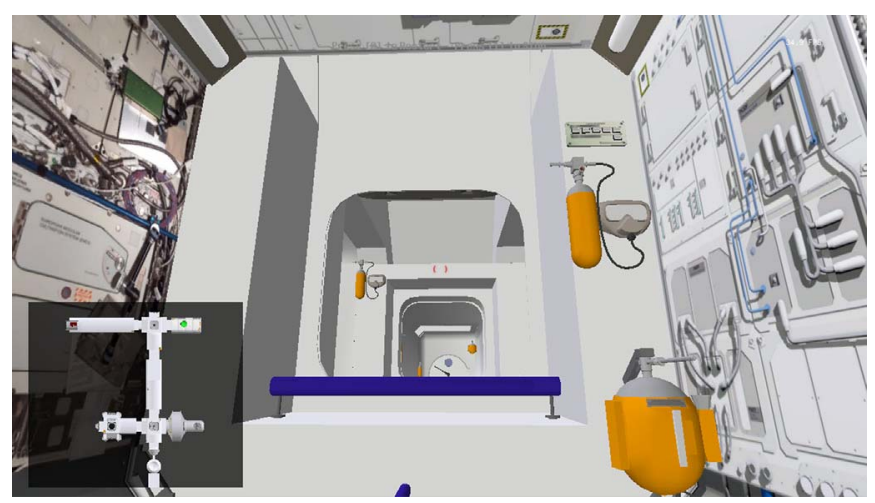

Fig. 8. A map of the ISS is integrated in the display of the trainee (from [15]).
Table 3

Currently implemented emergency equipment with interaction possibility.

- Press alarm button on corresponding panel

- Operate (close/open, latch/unlatch, usage as delta pressure indicator) the hatches

- Discharge Fire Extinguisher, also with nozzle in fire ports

- Wear Portable Breathing Apparatus (trainee's field of sight becomes obstructed)

- Perform CSA-CP measurement (including additional sampling probe SP 402) of combustion by-products

- Perform pressure measurements with Manovacumeter

green dot can be integrated in the view to ease the navigation through the station, as depicted in Fig. 8.

All emergency equipment was modeled to a somehow realistic but still simplified degree to allow some understanding of what the crew is working with in orbit, but since the learning objective is not the correct and detailed handling of this equipment, its operation was simplified and sometimes adapted in order to be better usable in the virtual environment. For example analog gauges of measuring devices were substituted with digital displays in order to enhance readability as the monitors and/or projectors which display the virtual world to the user would run at limited resolutions.

The trainee can pick up several equipment items but can only operate one at a time (with the exception of the portable breathing apparatus which can be used in conjunction with other items as well). He/she can choose which one to use by toggling through the stack of items by pressing a button on the game pad, see also Table 3.

\subsection{Control and evolution of the simulation scenario}

In the above mentioned computer network of the simulation environment it is also possible to include a PC which is then configured with an instructor display. This allows an instructor to follow the ongoing simulation with some supplemental information (e.g. constantly updated pressure readings of all modules) and options to control the simulation. The emergency scenario can be selected (fire/rapid depress) as well as the location of the fire/the leak and the leak rate. In the fire case based on that input a mathematical algorithm calculates the values for the three "measured" combustion products $(\mathrm{CO}, \mathrm{HCl}, \mathrm{HCN})$ and constantly updates the values over time for all modules as well as for all fire port locations. It is up to the instructor to determine whether an equipment power down, a PFE discharge or a full module powerdown then kills the fire and ensures decreasing values.

For the depress case the leaking module, the current leak rate (influenced by the remaining pressure within the leaking module) and the open/close status of all hatches are the parameters of the algorithm, which calculates the current pressure for each module, which is then used as input for the Manovacumeter display the student can see.

\section{First experiences and further development}

Currently, the simulator is used in a stand-alone configuration for test purposes and to gain first-hand experience. The trainee is performing inside the CAVE whereas the instructor plays the part of the control center from outside the simulator. As a next step it is foreseen to introduce the simulator in the procedure walkthroughs of the emergency classroom training. Then the different roles can be shared among the student: one is playing the crew, one the control center(s) and others can follow the scenario from outside and comment or ask questions. 
The last step is then its usage during emergency simulations, in which a full Col-CC flight control team in the training control room interacts with the colleague playing the crew in the simulator. For that setup, some administrative and technical hurdles have to be overcome, because a data/video/voice link between the high security area of a control room and the public environment of the CAVE triggers some security concerns.

To use the simulator during emergency simulation it is required to connect the simulator CAVE with the control room intercom system, which then establishes the possibility of direct interactions between the student in the virtual environment and the Flight Controllers.

A video feed of the virtual scenario into the control room would also be an interesting option, but would not be mandatory however. This would also require the implementation of an avatar as the visual representation of the virtual astronaut. Since all Flight Control Team simulations are controlled, directed and observed from a dedicated simulation control room, this room also would need to be connected to the simulator network to allow the sim officers to also control and oversee the simulation running in the CAVE.

There is also ongoing work to link the 3D virtual simulator to a dedicated Columbus simulator [17], which generates Columbus telemetry for the control room displays and reacts on telecommands of the Flight Controllers. This way a Flight Controller playing the crew would immediately "see" the result of any ground action on the astronauts, e.g. that removing the power on dedicated channels would cause a turned off light on board/in the virtual world. Also commands sent by the astronauts via the corresponding laptops in the virtual world can influence the telemetry indicated on ground side.

A future usage in the DLR School lab [18] for educational purposes is also foreseen.

\section{Conclusion}

The novel concept of ISS emergency simulations for Flight Controllers using virtual reality increases the awareness of the ground personnel for the crew actions in an ISS emergency case, and the efficacy of emergency training is expected to grow by the more immediate "immersion" of the trainees in the scenario. The independency of emergency training events from certified astronaut support makes them more flexible and could help to increase their frequency.

\section{Acknowledgment}

The hardware was funded by the German Ministry of Economics and Technology (BMWi) under the funding code $50 \mathrm{JR}$ 0462 .

\section{References}

[1] G. Musgrave, A. Larsen, T. Sgobba, Safety Design for Space Systems, Elsevier, Amsterdam, 2009.

[2] J. Campan, T. Uhlig, D. Herrmann, Human spaceflight operations, in: T. Uhlig, F. Sellmaier, M. Schmidhuber (Eds.), Spacecraft Operations, Springer, Berlin, Germany, 2014.

[3] M. Barratt, Medical support for the International Space Station, Aviat. Space Environ. Med. 70 (1990) 155-161.

[4] R. Friedman, Fire safety in spacecraft, Fire Mater. 20 (1996) 235-243.

[5] An outdated version of the ISS Emergency procedures is available under 〈http://www.spaceref.com/iss/ops/iss.emergency.ops.pdf), (accessed 15.09.15).

[6] J.W. Kim, J.L. Crassidis, S.R. Vadali, A.L. Dershowitz, International Space Station Leak localization using vent Torque estimation, in: Proceedings of IAC55, 2004

[7] W.C. Wong, R. Guidry, D.M. Arneson, D. Zimmerman, M. Downing, V.A. Castro, D.L. Pierson, Biosafety onboard the International Space Station, Appl. Biosaf. 16 (3) (2011) 158-162.

[8] S.M. Duchesne, J.J. Sweterlitsch, C.H. Son, J.L. Perry, Impacts of an ammonia leak on the cabin atmosphere of the International Space Station, in: Proceedings of International Conference on Environmental Systems, American Institute of Aeronautics and Astronautics (ICES), 2012.

[9] M. Pinni, A. Bade, N. Illmer, R. Seine, Crew emergency training for the columbus module, in: Proceedings of IAC63, 2012.

[10] L. Ravagnolo, L. Winterling, R. Moss, R. Seine, M. Pinni, ATV emergency training, in: Proceedings of IAC63, 2012.

[11] T. Uhlig, K. Özdemir, D. Sabath, Training concept of the columbus flight control team, in: Proceedings of IAC62, 2011.

[12] W. Dick, L. Carey, J.O. Carey, The Systematic Design of Instruction, 7th ed., Allyn \& Bacon, Boston, 2011.

[13] B.S. Bloom, Taxonomy of Educational Objectives, Book I: Cognitive Domain, 2nd ed., Addison Wesley Publishing Company, United States, 1956.

[14] H.W. Reese, The learning-by-doing principle, Behav. Dev. Bull. 11 (2011).

[15] H. Helmholz, Entwicklung eines Virtual-Reality Trainings- und Simulationskonzeptes für die Columbus Flight Controller- und Astronauten-Ausbildung (Bachelor Thesis), University of Applied Science, Berlin, 2013.

[16] M. Fairchild, Enhancement of a Virtual Reality Training and Simulation Module for the Columbus Flight Control and Astronaut Training Program (Bachelor Thesis), Technical University of Munich (TUM), Germany, 2014.

[17] J. Denniston, T. Uhlig, The Columbus desktop trainer: an alternative solution to operations training, in: Proceedings of Space Ops Stockholm/Sweden, 2012

[18] D. Hausamann, T. Schüttler, D. Haigermoser, B. Kästner, The DLR school lab Oberpfaffenhofen - Attracting young people to science and engineering. Bridging the Gap between Research and Science Education, in: Proceedings of International Conference, Vienna, Austria, 2008. 